\title{
Neurobiological approaches to a better understanding of human nature and human values
}

GERALD HÜTHER*

\begin{abstract}
The most important finding made in the field of neurobiological research during the last decade is the discovery of the enormous experience-dependent plasticity of the human brain. The elaboration and stabilization of synaptic connectivity, and therefore, the complexity of neuronal networks in the higher brain centres depend to a far greater extent than previously believed on how - or rather, for which purpose - an individual uses his brain, the goals pursued, the experiences made in the course of his life, the models used for orientation, the values providing stability and eliciting a sense of commitment. The transmission and internalization of culture-specific abilities and of culture-specific values is achieved primarily during childhood by nonverbal communication (mirror neuron system, imitation learning) as well as by implicit and explicit experiences (reward system, avoidance and reinforcement learning). Therefore the structural and functional organization of the human brain is crucially determined by social and cultural factors. Especially the frontal cortex with its highly complex neuronal networks involved in executive functions, evaluation an decision making must be conceptualized as a social, culturally shaped construct.

The most important prerequisites for the transgenerational transmission of human values and their deep implementation into the higher frontocortical networks of the brains of subsequent generations are secure affectional relationships and a broad spectrum of different challenges. Only under such conditions, children are able to stabilize sufficiently complex networks and internal representations for metacognitive competences in their brains. This delicate process of experience-dependent organization of neuronal connectivity is seriously and often also persistently hampered or prematurely terminated by uncontrollable stress experiences. This danger ought be minimized by education programs aiming at the implementation of values of connectedness to others and to nature during the period of brain maturation.
\end{abstract}

Keywords: Neurobiology, synaptic connectivity, human nature, human values, transgenerational transmission, frontocortical networks, metacognitive competences.

\section{The unique plasticity of the human brain}

By far the most significant finding in the field of neurobiological
research in recent years is what we refer to today as experience-

* Gerald Hüther is Professor and head of the Neurobiological Research Unit at the Psychiatric Clinic of the University of Göttingen, Germany.

TD: The Journal for Transdisciplinary Research in Southern Africa, Vol. 2 no. 2, December 2006, pp. 331-343. 
dependent or use-dependent plasticity: It is the discovery that the neuronal and synaptic connections in the human brain can be altered. The complexity of these connections and their stabilization depend to a far greater extent than previously believed on how - or rather, for which purpose - an individual uses his brain, the goals pursued, the experiences made in the course of his life, the models used for orientation, the factors which provide emotional stability and a sense of commitment. All this is shaped by an individual's family relationships, the abilities passed on to him and the guiding ideals and the goals conveyed by the thoughts and aims, by the myths, legends and belief systems of the particular culture in which a person grows up. The neuronal connectivity patterns in the human brain are continuously adjusted to these social and cultural factors, at least in all those areas which are shaped and structured postnatally. In other words, the individual human brain in its mature state is a social construct.

Therefore, it is not astonishing that neuroscientists by their modern imaging techniques are able to identify numerous differences between the brains of differently encultured and socialized subjects at both, the structural and the functional level. These differences are most pronounced in the very slowly developing higher cortical association areas. Examples of such use dependent adaptive modifications and reorganizations of neuronal connectivity have been observed throughout lifetime, even in the brains of elderly subjects. The degree of brain plasticity is of course highest at younger ages, but the networks and connections between nerve cells can apparently be restructured and adjusted to new demands throughout lifetime. A prerequisite for such experience-dependent changes is the activation of the emotional centres (limbic system). This leads to the release of trophic, hormone-like substances, which stimulate the growth and the reorganization of nerve cell contacts and connections. Such emotional activation, i.e. situations which 'go deeply under the skin', are most often experimented during childhood and adolescence, but much less frequently during adulthood, when a person has learned to master almost all challenges of daily life as a routine.

The most important triggers for the adaptive modification and reorganization of neuronal networks and synaptic circuitry at any age are the problems encountered and the experiences made by an individual in the course of the so called stress-response. Therefore, the first part of this contribution will focus on the adaptive self-organization of neuronal connectivity through the mastery of challenges and of stressful experiences. In the second part, the influences of early affective relationships and of a culture of peace and non-violence on the developing brain will be somewhat closer examined. 


\section{Stress and the experience-dependent organization of neuronal connectivity}

Current stress research is characterized by fascinating insights into the mechanisms involved in the activation and the regulation of the neuroendocrine stress response and the consequences of this activation on the body and the brain. This progress is contrasted by a considerable degree of conceptual confusion. Until now, a generally accepted concept of stress is still elusive. Initially the term "stress" was used synonymous to "stressor" and no clear distinction was made between this stimulus and the reaction to it, the "stress-response".

This concept has now been replaced by the recognition that stimulus and response can not regarded as two independent, stable entities but rather represent two closely linked components mutually affecting each other in the course of the stress-reaction-process. This conceptualization explicitly implies important aspects, such as the character of the strain, the appraisal and the psychological as well as the emotional changes which occur in the course of this process. It implies that, if an individual is able to terminate a certain stressor by his own efforts, a controllable stress response is elicited, whereas an uncontrollable stress response is initiated when no adequate coping strategies are available or can be applied to terminate the stressor.

A controllable stress response is typically elicited when an individual has the subjective feeling that a certain demand or challenge can be met in principle by its own action but when this action is not (yet) ready, efficient or adequate enough to avoid the activation of his central stress responsive systems. The initial stages of the controllable and the uncontrollable stress response are identical. Both start with the recognition of a novel, unexpected, challenging or threatening stimulus which causes the generation of a non-specific pattern of arousal in the associative cortex and in the limbic structures. Through descending excitatory efferences, this activation is propagated to lower brain structures, especially to the central noradrenergic system. If the stressor is felt to be uncontrollable, the arousal of the higher cortical and limbic brain structures will not only persist but is even potentiated by the increased firing of noradrenergic afferences. Above a certain threshold, the sum of excitatory cortical and limbic, as well as of noradrenergic inputs to the neurosecretory hypothalamic nuclei will ultimately stimulate the release of corticotrophin releasing hormone and vasopressin, and thus, activate the HPA-system and stimulate adrenal glucocorticoid secretion. However, if the stressor is felt to be controllable, the non-specific pattern of arousal in the associative cortex will be funnelled into a specific activation of those neuronal pathways and circuits which are involved in the behavioural response to that stressor. Under these conditions, the enhanced noradrenergic output acts to facilitate the neuronal pathways activated in the course

TD, 2(2), December 2006, pp. 331-343. 
of this response. The reverbatory stimulation of the central stress responsive systems is no longer propagated, and the HPA-system is not at all or only slightly stimulated. Therefore, the controllable stress response may be regarded as an incompletely built up activation of the central stress responsive systems. It is characterized by a preferential activation of the central and the peripheral noradrenergic system.

Due to its extensive projections and the fact that adrenergic receptors are expressed not only by neurons but also by glial and endothelial cells, the central noradrenergic system is capable of modulating a great number of different brain functions:

Stimulation of neuronal adrenoreceptors increases the signal-to-noise ratio of cortical information processing, and contributes to the gating and to the facilitation of neuronal output patterns. Stimulation of adrenergic receptors of cerebral blood vessels leads to enhanced perfusion, increased brain glucose uptake and elevated energy metabolism. Activation of astrocytic adrenoreceptors stimulates glycogenolysis and the release of glucose and lactate as well as the formation and the release of various neurotrophic factors. Through these different effects, the increased noradrenergic output in the course of a controllable stress response contributes to the stabilization and facilitation of those neuronal pathways and connections which are activated in response to a certain controllable stressor. Repeated exposure to one and the same controllable stressor will thus lead to the successive facilitation of the neuronal circuitry involved in the behavioural responding. The noradrenaline-mediated stimulation of the synthesis and the release of neurotrophic factors by astrocytes will additionally favour structural adaptations through experience-dependent plasticity. Such stepwise adaptive modifications of the neuronal circuitry will automatically be triggered in the course of the controllable stress response until the original stressor can be adequately met by an efficient response. To some extent, this adaptive modification of associative cortical networks is comparable to catecholamine-mediated, peripheral structural adaptation processes, such as the increase of fur density in mammals upon repeated exposure to cold.

The particular importance of the repeated activation of noradrenergic neurons in the course of the response to controllable stress for central adaptation processes is further supported by the fact that specific mechanisms evolved in mammals which increase the output efficacy of the noradrenergic system in the course of future stress responses in individuals exposed to different kinds of controllable stressors. This up-regulation of noradrenergic activity upon exposure to different controllable stressors is seen at several levels: The firing rate of noradrenergic neurons increases, the synthesis, storage, and release of noradrenaline by noradrenergic nerve endings rises, and even axonal 
sprouting and intensification of noradrenergic innervation in certain brain areas, e.g. in the cortex, have been observed.

Evidently, controllable stress of very complex and diverse character is a prerequisite for the optimal expression of the individual's genetic potential and for the elaboration of a very complex neuronal circuitry in the brain. An impressive illustration of the complex and persistent effects of multiple experiences of many different controllable stress responses on brain structure and brain function are the influences of "enriched environments" on the development of the cortex of young experimental animals. Enriched environments provide many different stimuli which are novel and which can be explored. Rats which had grown up under such complex stimulatory environments show a thicker cortex, enhanced vascularization, elevated number of glial cells, enlarged dendritic trees of pyramidal neurons, and an increased density of synapses in the cortex. Additionally, in adulthood, they show diminished anxiety in novel environments and an increased response of their HPA-system under conditions of severe stress.

An uncontrollable stress response is elicited when the activation of the central stress sensitive systems cannot be terminated by an individual's own efforts, because his previously acquired strategies of appraisal and coping are not appropriate or can not be employed. Under such conditions, the initial arousal of cortical and limbic structures will persist and contribute to escalate the reverbatory activation of the central stress responsive systems culminating in the activation of the HPA-system and adrenal glucocorticoid secretion. Because of their lipophilicity, circulating glucocorticoids can easily enter the brain and bind to the glucocorticoid receptors expressed by neurons and glial cells. As in the periphery, it is their main function to attenuate the activation of immediate stress responsive systems and to prevent these initial reactions from overshooting.

However, glucocorticoids do not directly suppress the immediate central responses in the course of the stress response, e.g., the release of excitatory amino acids or of monoamines. Instead, most actions of glucocorticoids in the brain are delayed and involve changes at the level of gene expression. These alterations have longer-lasting consequences on neuronal and glial cell function and metabolism. Certain functions will be affected in a way such that the targets of the immediate stress response are better protected against the potential damage caused by an overshooting future activation. This is achieved at several different levels: through the suppression of c-AMP formation in response to adrenergic stimulation, through compromising cerebral energy mobilization or through the reduced formation of neurotrophic factors, growth of processes and synaptogenesis. Glucocorticoids have been shown to potentiate the glutamate-induced damage to neurons and their dendrites and are therefore able to interrupt the 
neuronal circuits involved in the initiation and propagation of the central stress response. The hippocampal pyramidal neurons are endowed with the highest density of glucocorticoid receptors and are therefore especially vulnerable to long-lasting elevations of circulating glucocorticoids caused by the uncontrollable stress. Also the noradrenergic axons and nerve terminals in the cortex appear to be particularly susceptible under such conditions and tend to retract and to degenerate. At the behavioral level, high concentrations of circulating glucocorticoids have been shown to facilitate the extinction of previously acquired reactions. The common feature of all these different effects caused by the activation of the HPA-system in the course of the uncontrollable stress response is their destabilizing influence on the previously established neuronal connectivity. The facilitation and stabilization of neuronal circuitry triggered in the course of previous controllable stress response is thus opposed, attenuated or even reversed in the course of an long-lasting uncontrollable stress. The destabilization of the previously established neuronal connectivity in cortical and limbic brain structures may lead to fundamental changes in cognition, emotion and behavior and, at least in the adult brain, may be a prerequisite for the acquisition of novel patterns of appraisal and coping and for the reorganization of the neuronal connectivity in cortical and limbic associative networks.

Throughout life, the repeated experience of the controllability of stressors is normally alternated by feelings of loss of control. The central adaptations resulting from the repeated exposure to controllable stressors are thus at least partly destabilized during periods when the loss of control is experienced. The activation of the central stress responsive systems by repeated experiences of controllable stress facilitates neuronal circuits and synaptic connections mainly through the activation of the central noradrenergic system. The neuroendocrine changes associated with the experience of uncontrollable stress, on the other hand, favour synaptic regression and the destabilization of previously established synaptic pathways and neuronal circuits. As long as the activation of the central stress system can be terminated by a cognitive, emotional or behavioural reaction, the neuronal circuits involved in this response become facilitated.

If no cognitive, emotional or behavioural responses are available to terminate the activation of the central stress response system, the underlying neuronal networks become destabilized. This may provide novel opportunities for the reorganization of neuronal circuits and the acquisition of novel coping strategies for a more efficient control of the novel environmental demands. But more often such destabilization processes pave the way into the manifestation of various kinds of psychopathologies.

The nature of what an individual considers life threatening, stressful 
challenges changes together with, and as a result of his improving sensory cognitive and intellectual realization of, and interaction with, the outside world. In infants, a stress response is initially only elicited in situations that demand the satisfaction of a basic need. Later, the central stress-responsive systems are most frequently activated by the recognition of certain social and cultural rules which prohibit the satisfaction of such a need. In the course of their socialization, individuals develop additional needs which are no longer basic but culturally acquired. The strategies which are chosen by an individual to meet each one of these challenges are strictly dependent on his previous experiences.

'Successful' behavioural strategies, i.e. the neuronal networks involved in the activation and execution of certain cognitive, emotional or behavioural reactions which make a certain type of stressor subjectively controllable, become increasingly reinforced and facilitated. Inadequate strategies which repeatedly fail to suppress and to silence the central stress responsive systems will either be eliminated or will become a constant source of dysregulation. By this self-optimization process, the cognitive, behavioural and emotional reactivity of an individual is fitted in a stepwise, trial-and-error manner to its changing environmental demands.

All newborns possess a certain repertoire of behavioural reactions which are activated in the course of, or together with, the activation of the central stress responsive systems when their homeostasis is threatened by cold, hunger, thirst etc. Thus, they all make the repeated early experience that their reactions are suited to terminate the central responses elicited by stressful experiences. This early recognition of the controllability of a stressor by an own action is one of the earliest associative learning experiences of a child and it has a strong imprinting impact on the developing brain. It is the prerequisite for the acquisition of an ever increasing repertoire of more and more specific and refined behavioural strategies for the control of stressors. This repeated experience of the controllability of stress is a prerequisite for the acquisition of behavioural strategies which allow an individual to act and not simply to react. The more successful these actions are, the more will the neuronal pathways and synaptic connections involved in a certain type of adaptive behaviour become strengthened and efficient coping skills for certain types of stressors be developed.

The ability to deal successfully with stressors strengthens the selfesteem and feelings of self-efficacy as much as the range of problemsolving skills of an individual. Consequently, the experience of the controllability of stress is the predominating experience and the driving force for the later development of those individuals of a social group which, within the socio-cultural and age-specific context of this 
group, will become the most successful, the most clever, but not necessarily the most flexible and the most stable ones.

Such personal qualities emerge already at rather young ages. They can only be developed on the basis of secure stable affectional relationships during early childhood and favourable temperamental attributes. It is important, that stressful experiences are encountered at a time and in a way that allows the feeling of the controllability of stress to increase through appropriate responses. Reinforcing interactions with and responses from other people are important prerequisites for the promotion of self-confidence and self-esteem. A child's ability to cope successfully with stress is therefore never due to the buffering effect of some supportive factor. Rather it is determined by the chain of sequential experiences made under the prevailing conditions of a given familial and socio-cultural context.

\section{The influence of early affectional relationships on brain development and behaviour}

Secure emotional relationships between the child and its caregivers are of uttermost importance for the integration of novel experiences into the already existing patterns of neuronal connectivity in the developing brain. When a new stimulus reaches the brain, it will elicit a certain kind of arousal in the associative networks. If this arousal pattern is identical with the pattern of arousal formed by the activation of already established neuronal connections (founded by earlier experiences), the new stimulus will be reorganized as already known and responded as usual, i.e., routineously. If the novel arousal pattern is at least a bit similar to the already existing patterns formed by earlier experiences, it may be integrated into these old patterns and will thus become stabilized as an extended, more complex pattern of neuronal activity. This is the way, how children (and adults) learn.

If this integration is impossible, because the novel arousal pattern is too strange and cannot be associated with any already existing pattern, the child may either neglect the challenge or - if the stimulus or the problem is large enough and does not disappear - activate an emergency reaction. All mammals process such very old, genetically programmed emergency reactions: fight, flight - and if neither the activation of one or the other does solve the problem - freezing. The latter is associated with a so-called uncontrollable stress response, which causes a massive and long-lasting release of stress hormones which may cause destabilization of already existing neuronal connections and hamper the formation of new ones. Under such condition of helplessness, nothing can be learned and already stored knowledge cannot be activated and the respective neuronal connectivity patterns may even get lost permanently. 
Secure emotional relationships provide the most potent protection against such overload and its consequences on the brain. They act to resilience the stress-system under conditions of massive arousal by too strange experiences or too strong stimulation. In the other extreme, when the novel stimulus is not very strong, secure emotional relationships act as 'emotional enhancers of arousal'. The child is thus encouraged and motivated in its attempts to realize and integrate a novel (otherwise too weak) stimulus. If such support by secure attachment relationships are not available to a child, it is easily either flooded by an overload of stimuli (no stable activation patterns can then be formed and integrated in the brain structures) or it is insufficiently aroused (and therefore no sufficiently strong activation patterns are build up and can be integrated in the brain).

Therefore, the quality of the relationship to its caregivers my either favour or hamper the acquisition of own knowledge and competence by the child and affect the complexity of the structural maturation of its brain in a beneficial or detrimental manner.

It is during the first three years of life when the vast majority of synapses is produced. The number of synapses increases with astonishing rapidity until about the age three years and then holds steady throughout the first decade of life. A child's brain becomes superdense, with twice as many synapses as it will eventually need. Brain development is, then, a process of pruning, i.e. use-dependent structuring. This is why early experience is so crucial: those neuronal networks and synaptic pathways that have been stabilized by virtue of repeated early experience tend to become permanent; the synapses that are not used often enough tend to be eliminated. In this way early experiences - positive or negative - have a decisive impact on how the brain is wired.

Compared to other primates, the maturation of the human brain, especially of the higher frontocortical brain regions is enormously prolonged in our species. It reaches a much higher degree of complexity and is much more affected by early experiences, by use- and disusedependent plasticity. The most delicate neuronal networks of the frontal cortex are the sites where the most complex, most sophisticated and the most human-specific brain functions will be generated: goal-oriented behavior and motivation, self-concept and self-efficacy, impulsecontrol, consciousness and the ability to transcend own thoughts and intentions into larger contexts. Also the ability to feel what others feel, and to experience feelings of connectedness, peace and love are generated by the most intricate neuronal networks located in the frontal (frontoorbital) cortex. These networks and the abilities mediated by them are not preformed by an inherited genetic program. They all must be acquired, stabilized and facilitated by experience dependent plasticity, i.e., by education, socialization and enculturation. 
Genetically driven are only the enormous offerings made in individual brain areas (including the frontal cortex) at certain periods in the form of an overproduction of neuronal dendritic and axonal processes and an overabundance of synaptic contacts (critical periods). How many and which of these offerings can be maintained and become integrated into larger functional networks is dependent on their stabilizing inputs, i.e. the complexity of experiences made by a child during these early critical periods of brain development. But the most complex and most slowly developing neuronal networks in the frontal cortex are not only vulnerable to the lack of stabilizing inputs. They are at least as vulnerable to overstimulation and to the destabilizing influences mediated by ascending projections from subcortical (limbic, hypothalamic and brain stem) stress-sensitive systems. The enduring activation of an uncontrollable stress response will seriously hamper and suppress the elaboration and stabilization of the complex neuronal and synaptic connections in higher cortical association areas.

If a child experiences constant stress and anxiety, e.g. from not being able to structure and understand the world around it, or from the absence of grown-ups relieving the anxiety, the structuring of the brain risk be destabilized and regressing. Instead of structures enabling problem solving, the experience of own inability and incompetence is then structurally anchored in the developing brain. The part of the brain that is particularly open to the outside influences is the frontal cortex in which the experineces of learning and socialisation are structured.

\section{A neurobiological view on the prerequisites for a culture of peace and non-violence}

The development of the human brain is much less (pre-)determined by genes than previously thought. Our brain is a self-organizing, open system, characterized by an enormous degree of experience-dependent plasticity. Especially in the higher brain regions, the connections between nerve cells are structured by use and disuse, i.e. by activity patterns which are generated in the brain by sensory inputs. The more complex the situations we learn to deal with, the more sophisticated are the experiences we make, and the larger will be the degree of connectivity formed between the nerve cells in our higher brain centres.

When novel demands cannot properly responded by the activation of an already established pattern of connectivity in the higher brain regions, emotional centres in the midbrain become activated. Without this activation new experiences cannot be made and anchored in the brain structures. The stronger the activation, the more firm the structure. E.g. when parents play 'hide and seek' with their baby, the baby 
feels anxiety because the parent disappear and it does not know how to rectify the situation. When the parent reappears the anxiety is relieved. When the baby experiences this again and again a firm structure is formed from the experience that 'my parent is able to relieve my anxiety and I will be fine'. This structure is stored in the brain enabling the child to face future situations with anxiety with the experience that 'if I involve my parent the anxiety disappears', i.e. a problem solving skill is learned, enabled by brain structures.

It is paramount for the development of the brain (and thus for the ability to internalize emotional, social and intellectual knowledge) that the child is surrounded by grown-ups that help it relieve anxiety and stress by teaching it how to master problem solving in challenging situations thus creating new experiences. Since the brain cannot stimulate itself to develop the needed functionality, and the child wont know how to, the child depends on the grown-ups around it to provide the stimuli. Secure emotional relations with the primary care takers are therefore so important.

Animal experiments have shown that even newborn rats, raised by a 'bad' or less 'competent' mother, will themselves become 'poorly gifted' mothers, even if they were born by a 'good' mother. Parental competence is obviously not inherited but acquired already in rats and definitely even more so in humans. Here, parents must provide almost every competence, skill and cultural achievement to their children. Without their example, children would not even learn to stand upright or to speak. The better the competences of the parents, the greater the chance that they will be transmitted from one generation to the next. However, this process is only efficient if the parents are also able to engage emotionally in their relation to the child. This ability is also largely determined by their personal experiences with emotional relationships during early childhood.

During early childhood, aversive or insecure attachment relationships are the most important trigger for the activation of uncontrollable stress-responses. Therefore, insecurely attached children are unable to develop a highly complex neuronal and synaptic connectivity in their brain, especially in the frontal cortex. They have difficulties to acquire a broad spectrum of different coping strategies, to maintain a high level of creativity and curiosity, to constructively interact with others, and to develop feelings of connectedness, love and peace. Instead such emotionally labile children will tend to use and facilitate various less sophisticated, pseudo-autonomous, egocentric and even autistic behavioural strategies. They have difficulties to feel what others feel and to accept social rules. They are unable to control their impulses and they tend to various forms of violent destructive behaviour. Because of their poorly developed self-concept and their lack of self-efficacy, such children can easily be manipulated by 'strong 
others', e.g. a 'Führer'. Therefore all totalitarian regimes have always made special affords to systematically disrupt the formation of secure early attachment relationships between mothers and their children.

In order to prevent such negative effects of aversive early childhood experiences on later individual life and on the society, and to pave the way to a culture of peace and non-violence, particular efforts must be made to strengthen the relationship between parents and their children as early as possible, i.e. already during pregnancy, and to protect children against insecurity, anxiety and stress during early childhood. Since most parents are not aware of the sculpturing influences of their own relationships on the developing brains of their children, education programs may help and should be installed to overcome this deficit. These programs should aim to strengthen the affectional relationship between parents and their children, to inform the public about the negative influence of psychosocial and other stressors on brain development, and to help to prevent distortions of the relationship between parents and their children by aversive, traumatic or neglecting experiences. Particular efforts must be made to support secure attachment relationships and the feeling of emotional stability in our children, if we want to create a more peaceful world. Only then human subjects will have a chance to unfold their full potential.

Thus, modern brain research confirms an old Indian proverb: putting on pressure and inducing fear are not only unsuitable strategies for improving performance; they also strangle curiosity and creativity in schools, businesses and civil administration. It's high time to think about a new relationship culture. And slowly but surely, this is starting to be implemented as a successful leadership concept everywhere. This concept is called "Supportive Leadership", and it aims at the promotion of the readiness to achieve through support, recognition and encouragement. What should grow better has to be adequately watered and fertilised.

Unfortunately, the strange notion has set in the minds of many people that it is or indeed must be tiring to achieve, whether to learn a lot in school or to work successfully later. Therefore in school or at work, it is all too often attempted to improve achievement through reward and if this doesn't work, by threat of punishment.

These learning by rote methods appear at first glance to work quite well. But on closer examination and particularly in the long term, they turn out to be extremely problematical. Through these forced methods, the achiever senses a feeling: he or she feels that they are being put under pressure.

Neuronal patterns thus activated in the brain are then coupled and connected with all that required to attain the achievement concerned. Who goes through this once or even repeatedly, always feels, even at a 
later date, this same unpleasant feeling in his stomach, whenever he faces a similar challenge.

In order to get rid of this, one can only try to keep well clear of the work or even to seek an even bigger reward. The enthusiasm for achievement, the enthusiasm for learning and to do it your way is usually gone forever. The seedling shrivels, because it has been pulled instead of being watered.

\section{Bibliography and notes}

Note: This contribution is a comprehension and a framework constructed on the basis of numerous original articles that have been publishes in the field of brain research, developmental neurobiology and psychology in recent years. My theory is outlined in G Hüther, The compassionate brain, (Shambhala Publ. Boston, 2006).

For the sake of clarity, citations of original work and references to related articles are not included here. The following reviews may serve as a guidelines to original references:

Eisenberg, L.: The social construction of the human brain. Am. J. Psychiatry 152 (1995), 1563-1575.

Giedd, J.N., Blumenthal, J., Jeffries, N.O. et al.: Brain development during childhood and adolescence: a longitudinal MRT study. Nature Neuroscience 2(10) = (1999), 861-863.

Hüther, G.: The central adaptation syndrome. Progress in Neurobiology 48 (1996), 569-612.

Hüther, G.: Stress and the adaptive self-organization of neuronal connectivity during early childhood. Int. J. Devl. Neuroscience, 16 (1998), 297-306.

Hüther, G., Doering, S., Rüger, U., Rüther, E., Schüssler, G.: The stress reaction process and the adaptive modification and reorganization of neuronal networks. Psychiatry Research (1999), 83-95.

Liu, D., Diorio, J., Day, J.C., Francis, D.D., Meaney, M.J.: Maternal care, hippocampal synaptogenesis and cognitive development in rats. Nature Neurosci, 3 (2000), 799-806. 